\title{
Stability of a crystallizing layer within a magma ocean
}

\author{
JULIEN MONTEUX ${ }^{1}$, BARAA QADDAH ${ }^{2}$ AND DENIS \\ ANDRAULT $^{3}$
}

${ }^{1}$ CNRS, Université Clermont Auvergne

${ }^{2}$ Ecole Centrale Supélec

${ }^{3}$ Université Clermont Auvergne

Presenting Author: julien.monteux@uca.fr

After the Moon Forming Impact and the subsequent largescale mantle melting event, a major chemical differentiation occurred within the primitive mantle. This differentiation is responsible for the separation between compatible elements that prefer the solid phase (e.g., $\mathrm{Mg}, \mathrm{Cr}$ ) and incompatible elements which prefer the liquid phase (e.g., Al, Na, Fe). Thus, upon cooling, the composition of the solid and liquid phases evolved away from a "primitive" mantle composition. The chemical difference between the two can lead to a density contrast and, hence, to a separation of the two phases, by sedimentation of crystals in the magma for example. The buoyancy of the molten phase is governed by the ability of heavy elements, in particular iron, to integrate the solid fraction. Although corresponding partition coefficients have been studied in a wide range of $\mathrm{P} / \mathrm{T}$, major uncertainties remain and the geodynamical and geochemical mechanisms of primitive mantle segregation are poorly constrained.

As the vigor of the thermal convection in the magma ocean tends to prevent the sedimentation of the solid grains, a detailed understanding of the overall dynamical behavior of this solid phase flow segregation is required. We have developed numerical models using COMSOL Multiphysics to monitor the chemical and thermal evolution of a convecting and crystallizing magmatic reservoir. These models solve the Navier-Stokes and heat transfer equations considering the Euler-Euler method to monitor the dispersed multiphase flow. Our study focuses on the vicinity of the solid boundaries where the flow is less turbulent and gradually slows down until the local flow speed becomes comparable to the settling speed of the crystals. The Rayleigh numbers investigated in our study range between $10^{6}$ and $10^{8}$. We also model the compatible/incompatible elements transfers by implementing a time dependent density contrast between the mineral phase and the residual liquid phase. Our models test the mantle crystallization dynamics for either positive or negative buoyancy of the molten material and show that the ability of the crystal fraction to disperse within the domain is strongly dependent on the crystal size, the density contrast and the magma viscosity. 